\title{
A Simple Atmospheric Pressure Ionization Source Coupled to a Time- of-Flight Mass Spectrometer for Breath Analysis
}

\author{
Jens Langejuergen ${ }^{*}$, Philipp Cochems, Stefan Zimmermann \\ Leibniz University Hannover, Appelstr. 9a, 30167 Hannover, Germany
}

\begin{abstract}
The analysis of exhaled breath accesses a variety of new diagnostic methods. However, this requires new analytical tools combining sufficient separation power (mass resolution) with ppt-level detection limits. A time-of-flight mass spectrometer (ToF-MS) is a suitable choice to analyze an entire mass spectrum of gaseous samples within a comparatively short sample time $(0.5 \mathrm{~s})$ at high mass resolution. However, the required detection limits can only be obtained by using atmospheric pressure ionization known from ion mobility spectrometry. Here we present a simple atmospheric pressure ionization source based on electron impact ionization coupled to a Bruker micrOTOF II mass spectrometer giving excellent mass resolution combined with ultra-high sensitivity. We achieve detection limits, e.g. for Acetone, in the lower ppt-range at $0.5 \mathrm{~s}$ integration time. The mass resolution is approx. 3000 at $60 \mathrm{u}$. However, one major drawback of this method is that competing proton transfer processes in the ionization chamber make quantitative analysis difficult. Thus, we use a gas chromatograph for pre-separation. As an example, here we analyze end-tidal human breath sampled on Tenax TA adsorption tubes using our $\mathrm{CO}_{2}$-triggered breath sampling unit. Furthermore, a thermal desorption system (TDS) coupled to a cold injection system (CIS) for sample injection into the gas chromatograph (GC) is used. Two-dimensional heat-maps of the abundance of ion masses versus the GC retention time are shown for inhaled (laboratory air) and exhaled human breath. Several substances are detected within the breathing gas sample only. An identification of these substances as well as a better control of the ionization process are subject to future investigations. Here we just present our tool for breath analysis.
\end{abstract}

\section{Introduction}

Since many volatile organic compounds, present in exhaled breath, are metabolic products, breath analysis has great potential in the clinical environment as a non-invasive method for diagnostics. However, identifying potential biomarkers in real-time at ppt-level concentrations requires new analytical methods having sufficient analytical power. In [1] Amann and Smith give an excellent overview of different technologies used in breath analysis today. Here we present a simple atmospheric pressure ionization source coupled to a Bruker micrOTOF II mass spectrometer giving excellent mass resolution combined with ultra-high sensitivity [2]. We use an atmospheric pressure ionization source (API) based on electron impact ionization as known from ion mobility spectrometry [3]. With this method we achieve detection limits, e.g. for Acetone, in the lower ppt-range at $0.5 \mathrm{~s}$ integration time. First results show that direct sampling of exhaled breath is possible. However, one major drawback of this method is that competing proton transfer processes in the ionization chamber make quantitative analysis difficult. Thus, pre-separation by gas chromatography is still necessary. Therefore, better control of the ionization process is one aspect of further investigations.

\section{Experimental Setup Sample Acquisition}

In fig. 1 the experimental setup for breath sample acquisition (active breathing) is shown. It is important to note, that our breath sampling unit $(24 \times 24 \times 15 \mathrm{~cm})$ is also suitable for clinical applications (ventilated 
patient). It is controlled by a LabView software to extract a small portion of the end-expiratory breath. Therefore, we monitor the $\mathrm{CO}_{2}$-level within the breath with a fast $\mathrm{CO}_{2}$-Sensor from Dräger as used already in other clinical applications. If the $\mathrm{CO}_{2}$-level is above a threshold (e.g. $2 \%$ ) $100 \mathrm{sccm}$ gas is pumped through a Tenax TA adsorption tube. The actual flow and the total amount of sampled gas are monitored. The acquisition stops automatically when a total amount of $500 \mathrm{ml}$ is sampled. The tubes (Supelco 28281-U) containing the adsorbed gases are then transferred to a thermal desorption unit. This makes it possible to perform bed-side sample acquisition while using laboratory instrumentation to analyze breathing gas of ventilated patients.

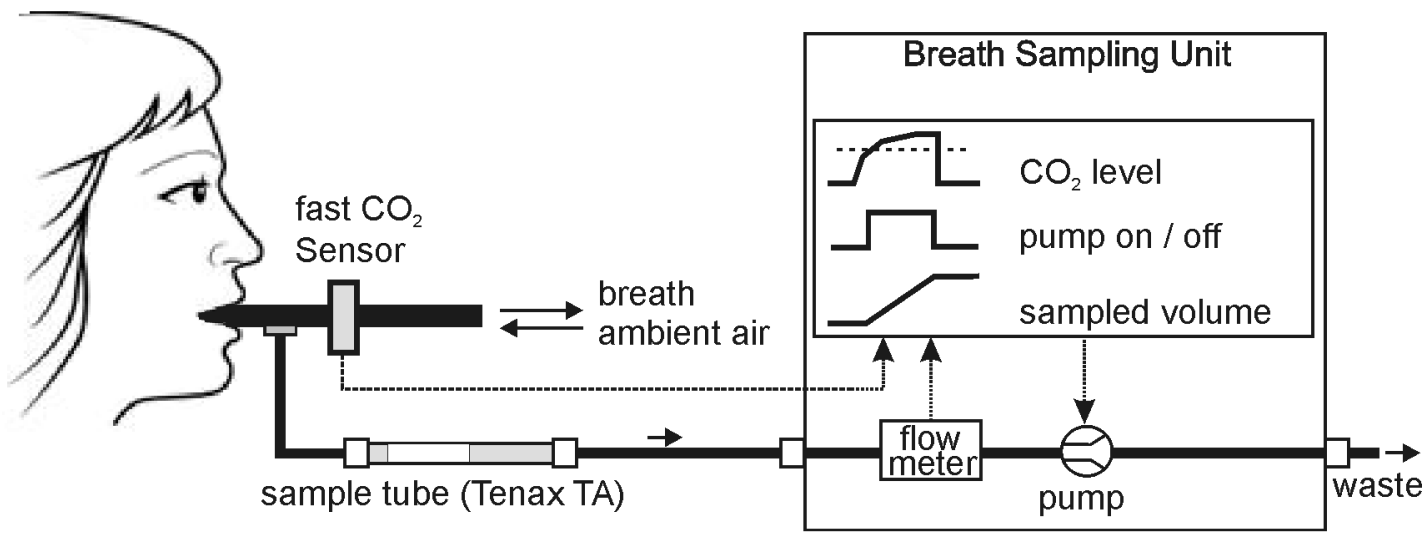

Figure 1 Breath sampling unit for Tenax TA adsorption tubes. A fast $\mathrm{CO}_{2}$-Sensor is used to monitor the $\mathrm{CO}_{2}$-level within the exhaled breath. If a level above a threshold (e.g. $2 \%)$ is detected a small portion $(100 \mathrm{sccm})$ of the endexpiratory gas is pumped through the adsorption tubes until the $\mathrm{CO}_{2}$-level decreases below the threshold value. The actual flow is monitored and the acquisition stops when a total amount of $500 \mathrm{ml}$ of end-expiratory breath is sampled.

\section{Sample Injection and Separation}

In fig. 2 the experimental setup for sample injection into the GC (Agilent 7890) is shown. The adsorption tube is placed into the thermal desorption system (Gerstel TDS) where it is heated up to $200{ }^{\circ} \mathrm{C}$ for $10 \mathrm{~min}$. A constant flow of $40 \mathrm{sccm}$ Nitrogen flows through the tube. $6 \mathrm{sccm}$ of this gas are directed through the cryo trap which is cooled by liquid nitrogen to $-85^{\circ} \mathrm{C}$. As cryo trap a glass tube of approx. $1 \mathrm{ml}$ volume filled with approx. $0.5 \mathrm{ml}$ of Tenax TA is used. After $10 \mathrm{~min}$. the cold injection system (CIS) is heated up to $200{ }^{\circ} \mathrm{C}$ with a rate of $12 \mathrm{~K} / \mathrm{s}$ and the desorbed gas is injected into the gas chromatograph (GC) at a flow of $6 \mathrm{sccm}$. The initial $\mathrm{GC}$ temperature is $40{ }^{\circ} \mathrm{C}$. It is increased to $200^{\circ} \mathrm{C}$ after $4 \mathrm{~min}$. of acquisition. The $\mathrm{GC}$ column is a 30 meter RTX-Volatiles (Restek) with an inner diameter of $530 \mu \mathrm{m}$ and a film thickness of $2 \mu \mathrm{m}$. The different components of the sampled gas are diluted by approx. $600 \mathrm{sccm}$ of dry air and then ionized in our atmospheric pressure ionization (API) source. The ionized sample gas is then transferred via gas flow and electric fields into the mass spectrometer.

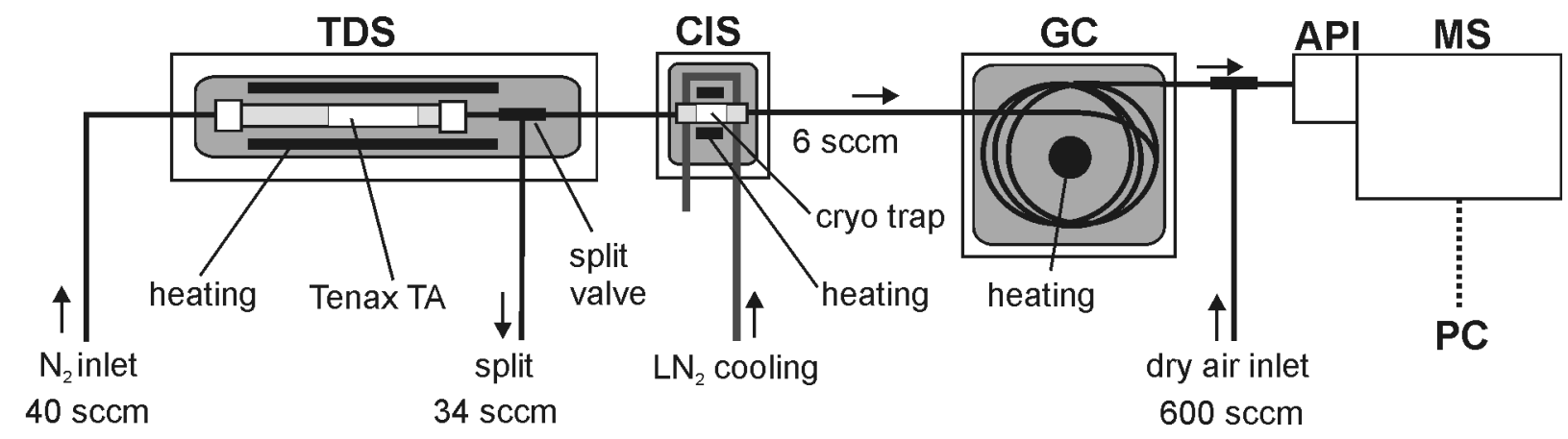

Figure 2 Experimental setup for sample injection into the gas chromatograph (GC). The desorption tube is placed into the thermal desorption system (TDS) where it is heated up. A constant flow of $40 \mathrm{sccm}$ Nitrogen flows through the tube. $6 \mathrm{sccm}$ of this gas are directed through the cryo trap. After $10 \mathrm{~min}$. the cold injection system (CIS) is heated up and the desorbed gas is rapidly injected into the gas chromatograph (GC) at a flow of 6 sccm. Past the GC the now separated substances are diluted by $600 \mathrm{sccm}$ dry air and ionized. 


\section{Ionization Source and GC-MS Coupling}

In fig. 3 the atmospheric pressure ionization source coupled to the time-of-flight mass spectrometer (ToF-MS) is shown. We are using a $300 \mathrm{MBq}$ Tritium (beta-decay) source giving free electrons with an average kinetic energy of $5.7 \mathrm{keV}$. Since Nitrogen has the highest concentration within the ionization source nitrogen ions are initially formed. Due to a chemical gas phase ionization process which is described in detail in [3] the neutral gas molecules leaving the GC are than ionized by charge transfer. The ionized gas is transferred by a gas flow and an electric field ( $\left.\mathrm{U}_{\text {Bias }}\right)$ into the MS. To ensure a sufficient gas flow into the MS an additional flow of $600 \mathrm{sccm}$ of clean dry air is provided by a zero-gas-generator. However, this dilution decreases the possible detection limit by a factor of 100 . Therefore, better control of the ionization process and transfer into the MS are aspects of further investigations. The ionized gas flows through a metallic transfer capillary which is coupled to the inlet capillary of the MS. Within the MS the ions are transferred and focused by different electrodes (ion optics). Due to the high electrical fields necessary to provide a sufficient ion transfer some analyte ions decay to fragments which than are too light to be detected. Due to this fragmentation some ions which are initially formed within the ionization source are not present in the mass spectrum. Furthermore, the minimum mass that can be detected is $21 \mathrm{u}$. However, API is a very soft ionization [2] which provides a less complex mass spectrum mostly containing the molecular peak and only few fragments.

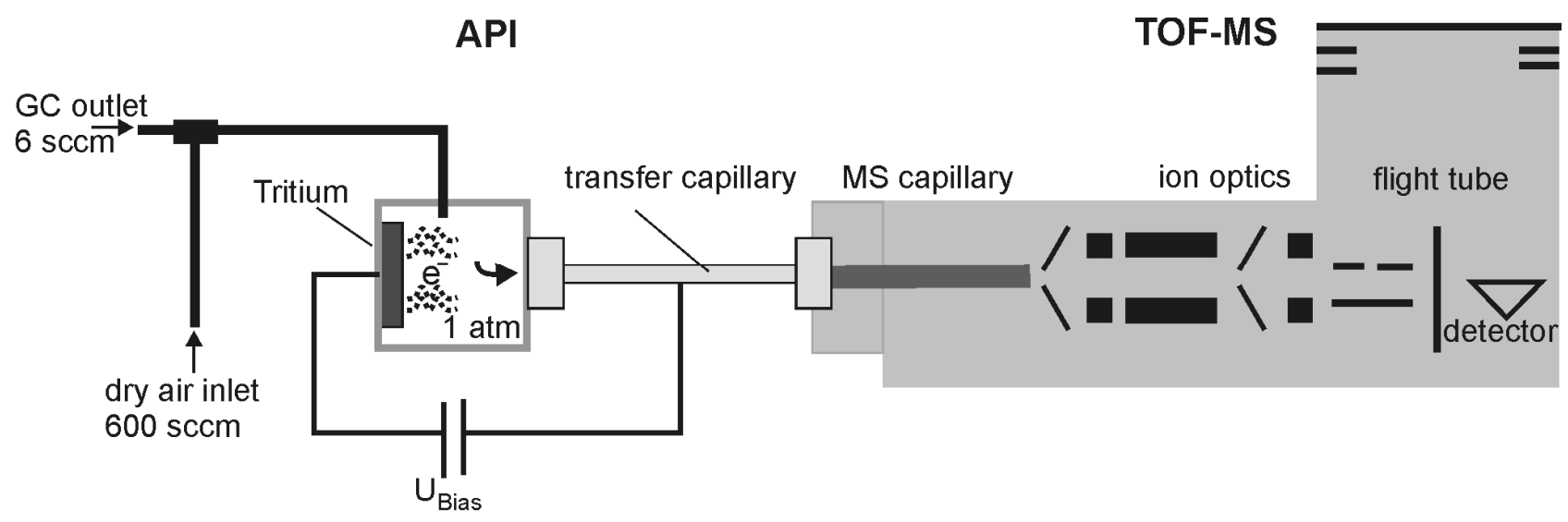

Figure 3 Atmospheric pressure ionization (API) based on electron impact. Electrons emitted from a $300 \mathrm{MBq}$ Tritium source are used. The gas flow out of the GC is diluted by $600 \mathrm{sccm}$ dry air from a zero-gas-generator.

\section{Experimental Results}

In fig.4 (left) the mass spectra of the sampled breathing gas is show at a $\mathrm{GC}$ retention time of 5:36 min. A dominant peak of protonated Acetone monomer at $59 \mathrm{u}$ and protonated Acetone dimer at $117 \mathrm{u}$ is visible. Due to the soft ionization no fragments are visible. The mass resolution $(\Delta \mathrm{m} / \mathrm{m})$ for both peaks is approx. 3000 which makes it possible to identify a single component even when multiple components with similar GC retention times are present. A MS sample time of $500 \mathrm{~ms}$ is used to resolve the different GC peaks. A number of $25 \mathrm{k}$ ions for the protonated Acetone dimer is detected which is three orders in magnitude above the noise level. On the left side of fig. 4 the mass spectrum at a GC retention time of 7:30 min. is shown. A protonated monomer and dimer of a substance not clearly identified yet are present at $87 \mathrm{u}$ and $173 \mathrm{u}$. We expect this substance to be Hexane.

In fig.5 the grayscale heat-maps for a conditioned desorption tube (left, zero-measurement), a breathing gas sample (center) and the inhaled laboratory air (right) are shown. The heat-maps show the mass spectrum (horizontal axis) versus the GC retention time (vertical axis). The brighter the spot in the heat-map the higher is the concentration of a certain mass at a certain time. The heat-map of the conditioned desorption tube (left) shows that no significant amounts of trace gases except Acetone at a GC retention time of 5:36 min. are present. Protonated water molecules $(19 \mathrm{u})$ which are expected to play a major role during the ionization 
process even at a dew point of $-66^{\circ} \mathrm{C}$ are not visible in the mass spectrum since the minimum mass which can be detected in our setup is $21 \mathrm{u}$.
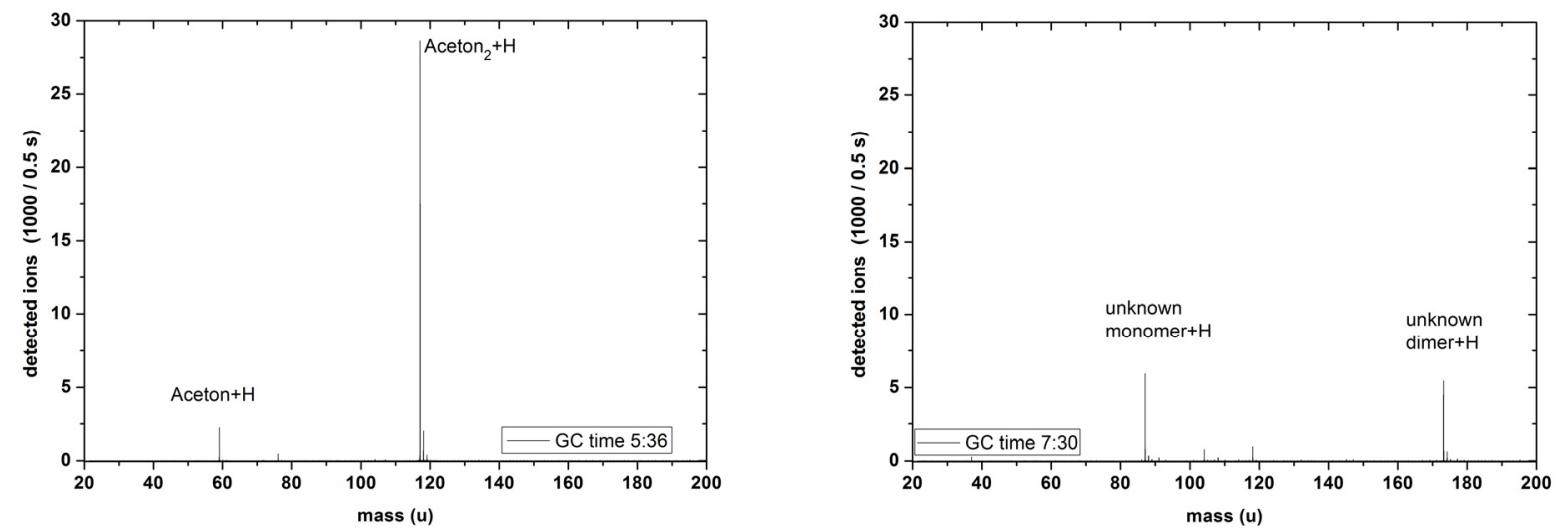

Figure 4 Mass spectra of breathing gas at different GC retention times. Protonated Acetone monomer and dimer are visible (left). One the right side the spectrum of a substance not clearly identified yet is visible at $87 \mathrm{u}$ and $173 \mathrm{u}$. It is expected to be Hexane.

The heat-map of a $500 \mathrm{ml}$ breathing gas sample (center) shows various substances - most of them not identified yet - which are not present in the laboratory air (right) or the conditioned desorption tube. Especially a significantly higher level of Acetone at a GC retention time of 5:36 min. is visible as expected. Surprisingly, no traces of Isoprene as found by other groups [4] using PTR-MS could be detected. We expect Isoprene either to be fragmented during ion transfer in the MS or to be discriminated by other substances with higher proton affinity and similar GC retention time.
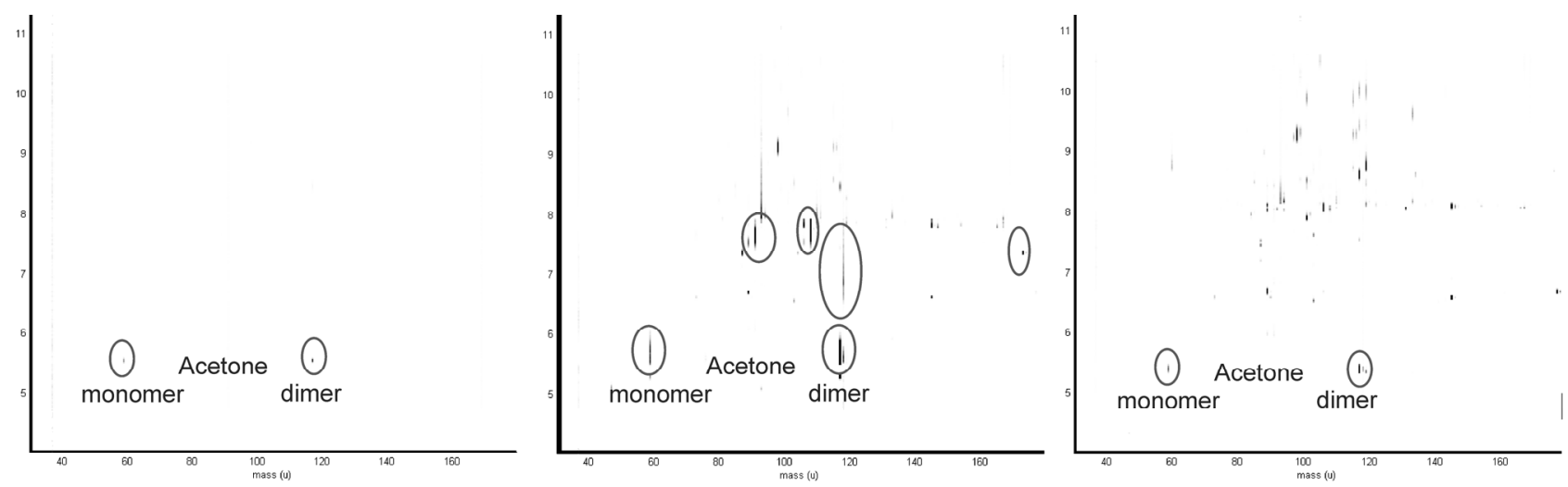

Figure 5 Grayscale heat-maps of the mass spectrum (horizontal) versus the GC retention time (vertical) for a conditioned desorption tube (left), a breathing gas sample (center) and the inhaled laboratory air (right) are shown.

\section{Literature}

[1] A. Amann, D. Smith, Breath Analysis for Clinical Diagnosis and Therapeutic Monitoring, World Scientific Publishing, 2005.

[2] J. Langejuergen, P. Cochems, S. Zimmermann, Pushing a Bruker micrOTOF II Towards ppq Detection Limits Using a Simple Atmospheric Pressure Ionization Source, Proc., XXV AHMT-Symposium, Shaker Verlag, 2011.

[3] G.A. Eiceman, Z. Karpas, Ion Mobility Spectrometry, CRC Press, 2005.

[4] W. Lindinger, A. Jordan, Proton-transfer Reaction Mass Spectrometry (PTR-MS): On-line Monitoring of Volatile Organic Compounds at pptv Levels, Chem. Soc. Rev. 27,347-375, 1998. 